\title{
The effect of different dosage of piascledine on hot flushes in postmenopausal woman of Jahrom, Iran.
}

\author{
Rasekhjahromi Athar ${ }^{1}$, Zareian Jahromi Mahshid ${ }^{2 *}$ Zarei Babaarabi Zahra ${ }^{3}$, Bigizadeh Shiva ${ }^{4}$, \\ Jamali Safieh $^{3}$ \\ ${ }^{1}$ Obstetrician and Gynecologist, Jahrom University of Medical Sciences, Jahrom, Iran \\ ${ }^{2}$ Jahrom University of Medical Science, Jahrom, Iran \\ ${ }^{3}$ Midwifery, Dr. Rasekh Clinic, Jahrom University of Medical Sciences, Jahrom, Iran \\ ${ }^{4}$ Statics, Jahrom University of Medical Sciences, Jahrom, Iran
}

\begin{abstract}
Introduction: Menopause is one of the natural stages of women's life usually begins in 45-55 y old. The most common symptom is hot flushes. The aim of this study is to evaluate the effect of different doses of piascledine on hot flashes of post-menopausal women.

Method: 69 postmenopausal women referred to Dr. Rasekh clinic was entered to the study and were randomly divided into 3 groups, first group; $300 \mathrm{mg}$ piascledine daily, second group; $300 \mathrm{mg}$ piascledine twice a day and third group; hormone replacement therapy $(1.25 \mathrm{mg}$ conjugated estrogen for $25 \mathrm{~d}$ and $10 \mathrm{mg}$ dydrogestrone for $15 \mathrm{~d}$ ). Hot flushing symptoms were evaluated before and after intervention using Blatt-Kupperman Menopausal Index (BKMI). The data were analysed by SPSS 21 software.

Result: BKMI had a reduction of 17.91 in first group, 20.9 in second group and 20.96 in third group. Conclusion: We found that a higher dosage of piascledine leads to a significant reduction in hot flushes rate and according to the effectiveness and also lack of side effects related to the higher dose of piascledine, it seems to be an appropriate alternative for hormone replacement therapy to treat the menopausal symptoms in high dosage.
\end{abstract}

Keywords: Hot flash, Menopause, Piascledine, Phytoestrogen. Abbreviations: HRT: Hormone Replacement Therapy; HR: Hormone Therapy; SSRI: Selective Serotonin Reuptake Inhibitors; CHD: Coronary Heart Disease.

\section{Introduction}

Menopause is a natural stage in woman's life begins between the ages of 45-55 with a mean age of incidence around 51 years worldwide [1]. During this time the ovaries produce lower amount of female hormones specially estrogen which leads to menopause symptoms [2]. At the first menstrual cycle becomes irregular then it completely stops. When a woman doesn't experience menstrual period for 12 consecutive months, she is regarded as a menopause woman [3]. Most women spend more than one-third of their lives after menopause [4]. Estrogen reduction leads to some physical and emotional changes such as night sweats, mood changes, vaginal dryness, tachycardia, insomnia and hot flashes [5]. These symptoms can be severe enough to negatively impact quality of life, work performance, and personal relationships [6]. Hot flashes have been reported as the most bothersome symptom of menopause accounting for $75 \%$ of symptoms [7]. 40 percent of premenopausal women, 70 to 80 percent of women who naturally go through menopause and 90 to 100 percent of those who have had ovarectomy, will experience hot flashes. Frequency of the hot flashes varies from woman to woman. In about 30 percent of the women it occur more than ten times a day [8]. Hot flashes start by a sudden redness of skin on the face, neck and chest and is associated with a feeling of intense heat in the upper body; it sometimes ends with extreme sweating and usually last about 1 to $5 \mathrm{~min}$ [9]. Hot flashes usually occur during the night so they affect sleeping patterns and lead to perspiration and sleeplessness. Definitely, insomnia causes anger, restlessness and reduces mental functions, hence makes the body vulnerable to all sorts of stress disorders and can be indirectly related to coronary heart diseases [8].

Menopause does not require any medical treatment itself, the treatment is usually used to relieve the symptoms and to prevent or reduce complications caused by the menopause [10]. Both hormonal and no hormonal treatment modalities are available for management of the mentioned symptoms. Hormonal therapy includes estrogen and combined estrogen/ progestin therapy. Non-hormonal therapy includes isoflavones, 
gabapentin, clonidine, selective Serotonin Reuptake Inhibitors (SSRI's), black cohosh, and vitamin E [11]. Hormone Therapy (HT) is the most effective treatment for severe postmenopausal symptoms [12]. Hormone replacement therapy is contraindicated for the prevention of cardiovascular disease in postmenopausal women [13], moreover, with HRT the incidence of endometrial cancer, breast cancer, stroke and venous thromboembolism could be increased [14]. Despite the effectiveness of HRT on hot flashes, today there are disagreements about this type of treatment due to the mentioned complications caused by long-term use. It has been demonstrated that women's attitudes towards the menopause and their knowledge of the benefits and risks of HRT have a direct effect on their use of HRT [15]. Common side effects of estrogen include breast soreness, which can often be minimized by using lower doses. Vaginal bleeding occurs in almost all women receiving cyclic estrogen-progestin regimens and is common in the early months of a continuous estrogenprogestin regimen [10].

On the other hand, hormone replacement therapy is forbidden for women with the following: a history of breast cancer, CHD (Coronary Heart Disease), a previous venous thromboembolic event or stroke, active liver disease, unexplained vaginal bleeding, high-risk endometrial cancer, or transient ischemic attack [10]. So in these cases HRT is not recommend and it's better to use other treatments instead. Phytoestrogens are polyphenols that are structurally similar to endogenous estrogen and have weak estrogenic properties [16]. It has a positive effect on the reduction of the symptoms related to the menopause like hot flashes and improves mood and quality of life in postmenopausal women [17]. A litany of health benefits including a lowered risk of osteoporosis, heart disease, breast cancer, and menopausal symptoms, are frequently attributed to phytoestrogens [18].

Nowadays, Soy foods and supplements have been the subject of much interest for the reduction of menopausal symptoms because of their high concentrations of phytoestrogens [19]. Piascledine is an herbal medicine derived from avocado and soy which is used to relieve the hot flash as menopausal symptoms in women [20]. This drug is made in France and has no side effects. The aim of our study was to evaluate the effect of different doses of piascledine on hot flashes in postmenopausal women To determine whether the higher dose of piascledine can be more effective in controlling hot flashes or not.

\section{Materials and Methods}

\section{Sampling}

This interventional study was approved by the Jahrom University of Medical Science Ethics Committee. The ethical code is Jums.REC.1393.121. This study was done from January 2011 to September 2012. After a complete explanation about the process of the study, a written informed consent was obtained from the patients so participation in this study was completely voluntary.
The patients were selected through convenience sampling and randomly divided into three groups. According to the previous researches, considering $\mathrm{CI}=95 \%$ and power $=80 \%$ the sample size in each group was determined 23, so the total sample size was 69.

\section{Study participants}

All the menopause women with hot flashes who referred to the Dr. Rasekh clinic whose diagnosis was confirmed by the Kuperman index were entered to the study. We regarded them as the menopause ones if they didn't experience any menstrual flow for a minimum of 12 months.

\section{Inclusion criteria}

The patients who had not used hormone therapy (except first group), dietary supplements and herbal medicines to relieve menopausal symptoms were entered to the study.

\section{Exclusion criteria}

The women who were not able to use or continue their drugs due gastrointestinal symptoms were excluded.

\section{Intervention}

The patients were randomly divided into three groups. The first group received $300 \mathrm{mg}$ piascledine once a day for 3 months. The second group received $300 \mathrm{mg}$ piascledine twice a day for 3 months and the third group received HRT $(1.25 \mathrm{mg}$ conjugated estrogen daily for $25 \mathrm{~d}$ and $10 \mathrm{mg}$ dydrogestrone daily for $15 \mathrm{~d}$ ). All three groups were evaluated 4 times based on the Kupperman Index: before the intervention, 1, 2 and 3 month after that.

\section{Questions}

The Kupperman index is a numerical conversion index including 11 menopausal symptoms: hot flushes (vasomotor), paresthesia, insomnia, nervousness, melancholia, vertigo, weakness, arthralgia or myalgia, headache, palpitations, and stinging. Each symptom on the Kupperman index was rated on a scale from 0 to 3 for no, slight, moderate, and severe complaints. To calculate the Kupperman index, the symptoms were weighted as follows: hot flashes $(4 \mathrm{X})$, paresthesia $(2 \mathrm{X})$, insomnia $(2 \mathrm{X})$, nervousness $(2 \mathrm{X})$, and all other symptoms (1X). The highest potential score is thus 51. This index has been used widely in the studies of menopausal symptoms so its validity is confirmed [23-25].

\section{Analysis}

The statistical analysis was performed with SPSS software ver. 16. We used Mauchlys and ANOVA test to evaluate the consistency of the statistical data ( $p$-value of $\leq / 001)$. 


\section{Results}

This study was conducted on 69 eligible women, who were divided into three groups ( 23 patients in each group). From the participated patients, 67 of them were married and 2 were single. They were aged between $35-69$ y old. The average age of the participants was $46.6 \pm 87 \mathrm{SD}$ (in terms of years). The average time from the last menstrual period was 19.5 $\pm 3.9 \mathrm{SD}$ (in terms of month). The average Body Mass Index (BMI) of the patients was $27.05 \pm 52 \mathrm{SD}$.

In general, from all three groups before the intervention, $21.7 \%$ were asymptomatic while $33.3 \%$ had severe symptom. But, at the end of the study all of the patients had no severe symptoms, $89.9 \%$ were asymptomatic, and mild symptoms of hot flashes remained in only $7 \%$ of patients.
We used Blat Kuperman scale to measure the symptoms of the menopause. So including factor of four, the average rate of hot flashes in the three groups before the intervention was $22.63 \pm$ 1.24 SD and at the end of the study it reached to $2.56 \pm 39 \mathrm{SD}$ with a significant reduction. all eleven variables related to each group (hot flashes, numbness, insomnia, nervousness, severe sadness, dizziness, weakness, muscle pain or arthritis, headaches, heart palpitations and tingling of the skin) had a significant decrease.

In the first group in which the patients had used one capsule of piascledine per day, the average number of Kuperman scale (including eleven variables) before and after intervention was $21.82 \pm 2.20$ and $7.69 \pm 1.33 \mathrm{SD}$ respectively which shows a significant reduction (Table 1).

Table 1. Comparison of BKM index before and after intervention in the first group.

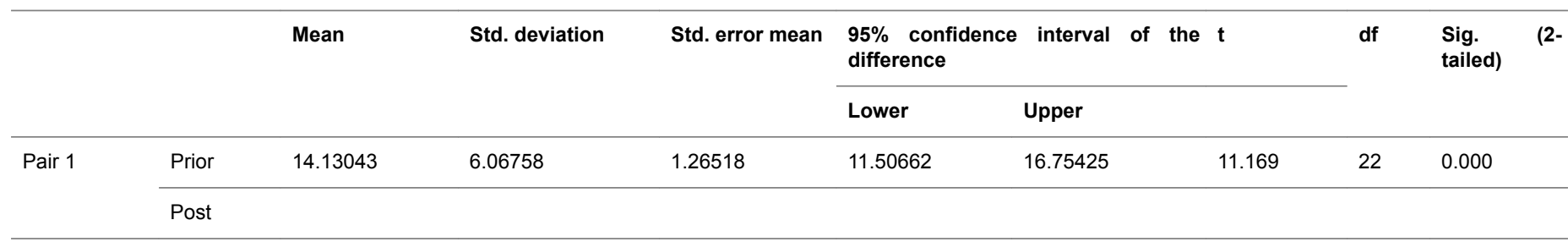

In the second group in which the patients had used two capsule of piascledine per day the average number of Kuperman scale before and after intervention was $24.69 \pm 1.95 \mathrm{SD}$ and $6.28 \pm$ $56 \mathrm{SD}$ respectively which shows a significant reduction (Table 2).

Table 2. Comparison of BKM index before and after intervention in the second group.

Mean N Std. deviation Std. error mean

\begin{tabular}{llllll} 
Pair 1 & Prior & 24.6957 & 23 & 9.39746 & 1.95951 \\
\cline { 2 - 5 } & Post & 6.2899 & 23 & 2.69924 & 0.56283
\end{tabular}

In the third group in which the patients had received hormone replacement therapy, the average number of Kuperman scale before and after intervention was $62 \pm 38 \mathrm{SD}$ and $21.39 \pm 2.29$ SD respectively which shows a significant reduction (Table 3 ).

Table 3. Comparison of BKM index before and after intervention in the third group.

\begin{tabular}{|c|c|c|c|c|c|c|c|c|c|c|}
\hline & & \multirow[t]{2}{*}{ Mean } & \multirow[t]{2}{*}{ Std. deviation } & \multirow[t]{2}{*}{$\begin{array}{l}\text { Std. } \\
\text { mean }\end{array}$} & \multirow[t]{2}{*}{ error } & \multicolumn{2}{|c|}{$\begin{array}{l}95 \% \text { Confidence interval of the } \\
\text { difference }\end{array}$} & \multirow[t]{2}{*}{$\mathbf{t}$} & \multirow[t]{2}{*}{ df } & \multirow[t]{2}{*}{ Sig. (2-tailed) } \\
\hline & & & & & & Lower & Upper & & & \\
\hline Pair 1 & Prior & 20.76812 & 11.43644 & 2.38466 & & 15.82263 & 25.71361 & 8.709 & 22 & 0 \\
\hline
\end{tabular}

According to ANOVA test, the rate of hot flushes related to each group was not significantly different before the intervention. But after three months of intervention, the rate of hot flushes of third group (who received hormone replacement therapy) was significantly different compared to first and second groups (P-value $\leq 0.05$ ). Reduction in the rate hot flashes was not significantly different between the first and second groups (Table 4).

Table 4. Comparison of the hot flashes between 3 groups.

\begin{tabular}{|c|c|c|c|c|c|c|c|}
\hline \multirow[t]{2}{*}{ Dependent variable } & \multirow[t]{2}{*}{ (I) Drug } & \multirow[t]{2}{*}{ (J) Drug } & \multirow{2}{*}{$\begin{array}{l}\text { Mean difference (I- } \\
\text { J) }\end{array}$} & \multirow{2}{*}{ Std. error } & \multirow[t]{2}{*}{ Sig. } & \multicolumn{2}{|c|}{$95 \%$ confidence interval } \\
\hline & & & & & & Lower bound & Upper bound \\
\hline Sum q10 & Pias 1 in every day & Pias 2 in every day & -2.86957 & 3.05368 & 0.351 & -8.9664 & 3.2273 \\
\hline
\end{tabular}




\begin{tabular}{|c|c|c|c|c|c|c|c|}
\hline & & & & & & & \\
\hline & & Srerojen & 0.43478 & 3.05368 & 0.887 & -5.6621 & 6.5317 \\
\hline & \multirow[t]{2}{*}{ Pias 2 in every day } & Pias 1 in every day & 2.86957 & 3.05368 & 0.351 & -3.2273 & 8.9664 \\
\hline & & Srerojen & 3.30435 & 3.05368 & 0.283 & -2.7925 & 9.4012 \\
\hline & \multirow[t]{2}{*}{ Srerojen } & Pias 1 in every day & -0.43478 & 3.05368 & 0.887 & -6.5317 & 5.6621 \\
\hline & & Pias 2 in every day & -3.30435 & 3.05368 & 0.283 & -9.4012 & 2.7925 \\
\hline \multirow[t]{6}{*}{ Mean q1, q2, q3 } & \multirow[t]{2}{*}{ Pias 1 in every day } & Pias 2 in every day & 1.4058 & 1.22478 & 0.255 & -1.0395 & 3.8511 \\
\hline & & Srerojen & $7.07246^{\star}$ & 1.22478 & 0 & 4.6271 & 9.5178 \\
\hline & \multirow[t]{2}{*}{ Pias 2 in every day } & Pias 1 in every day & -1.4058 & 1.22478 & 0.255 & -3.8511 & 1.0395 \\
\hline & & Srerojen & $5.66667^{*}$ & 1.22478 & 0 & 3.2213 & 8.112 \\
\hline & \multirow[t]{2}{*}{ Srerojen } & Pias 1 in every day & $-7.07246^{*}$ & 1.22478 & 0 & -9.5178 & -4.6271 \\
\hline & & Pias 2 in every day & $-5.66667^{*}$ & 1.22478 & 0 & -8.112 & -3.2213 \\
\hline
\end{tabular}

*The mean difference is significant at the 0.05 level.

Then, the differences in the numbers of Kuperman scale were determined in all three groups before and after the intervention. Accordingly, the reduction of symptoms in both groups using piascledine was very sensible. However, the difference between these two groups is not significant. Meanwhile, the difference between the groups taking piascledine and the group receiving hormone replacement therapy was significant. While the reduction rate of symptoms related to the second and third groups is not meaningful (Table 5).

Table 5. Comparison of the mean differences of Blatt_Kuperman index between 3 groups.

\begin{tabular}{|c|c|c|c|c|c|c|}
\hline \multirow[t]{2}{*}{ (I) drug } & \multirow[t]{2}{*}{ (J) drug } & \multirow{2}{*}{$\begin{array}{l}\text { Mean difference (I- } \\
\text { J) }\end{array}$} & \multirow{2}{*}{ Std. error } & \multirow[t]{2}{*}{ Sig. } & \multicolumn{2}{|c|}{$95 \%$ Confidence interval } \\
\hline & & & & & Lower bound & Upper bound \\
\hline \multirow[t]{2}{*}{ Pias 1 in every day } & Pias 2 in every day & -4.27536 & 2.69626 & 0.118 & -9.6586 & 1.1079 \\
\hline & Srerojen & $-6.63768^{*}$ & 2.69626 & 0.016 & -12.0209 & -1.2544 \\
\hline \multirow[t]{2}{*}{ Pias 2 in every day } & Pias 1 in every day & 4.27536 & 2.69626 & 0.118 & -1.1079 & 9.6586 \\
\hline & Srerojen & -2.36232 & 2.69626 & 0.384 & -7.7456 & 3.0209 \\
\hline \multirow[t]{2}{*}{ Srerojen } & Pias 1 in every day & $6.63768^{*}$ & 2.69626 & 0.016 & 1.2544 & 12.0209 \\
\hline & Pias 2 in every day & 2.36232 & 2.69626 & 0.384 & -3.0209 & 7.7456 \\
\hline
\end{tabular}

*The mean difference is significant at the 0.05 level.

\section{Discussion}

Understanding menopause-associated pathophysiology and developing new strategies to improve the treatment of menopausal-associated symptoms is an important topic in the clinic [21]. Both hormonal and non-hormonal treatments are available for management of the symptoms. There are few studies which have shown the disadvantages of HRT in menopause women. The study of Schumacher et al. conducted in 2003, shows that Estrogen plus progestin therapy increased the risk for probable dementia in postmenopausal women aged $65 \mathrm{y}$ or older [22].

Kim et al. worked on the effect of complementary and alternative therapy on the climacteric period. They find out that hormone replacement therapy increases the potential risk of thrombosis, cerebral infarction and breast cancer [23]. They also mentioned that finding a new alternative for HRT in management of menopause symptoms is an important concern of nowadays.

Epidemiological studies have shown that some of the diseases are more common in the West (including breast, endometrial, colon and prostatic cancers) which are less visible in East Asia that is due to consumption of phytoestrogens such as soy [24]. In a study conducted by Rua et al. it reveals that phytoestrogens appear to reduce the frequency of hot flushes in menopausal women, without serious side-effects [25].

Piascledine is an herbal medicine derived from avocado and soy and almost all the related researches to the piascledine has concentrated on its effects on arthritis, rheumatoid arthritis and osteoarthritis [26-29]. Laurent et al. worked on the relationship between osteoarthritis and nutrition, they have used two different doses of piascledine, 300 and $600 \mathrm{mg}$ per day [30]. 
In the Appelboom et al. study also which conducted in 2011 on the people with Knee Osteoarthritis and they have used two different doses of piascledine, 300 and $600 \mathrm{mg}$ per day [31]. So the use of piascledine twice a day is permitted and it has no side effects.

Only in one study conducted by Panahi et al. the effects of conventional HRT on the treatment of hot flush was compared with piascledine, they also evaluate the safety of piascledine in relieving postmenopausal symptoms. The patients in that open label clinical trial, randomized to receive piascledine capsule 1 $\mathrm{mg}$ or HRT $(0.625 \mathrm{mg}$ oral daily conjugated estrogen tablets, plus $2.5 \mathrm{mg}$ continuous oral daily medroxy progesterone acetate tablets) for 2 month. They revealed that due to low HRT compliance and its possible risks in long period of time and considering the same activity of soybean supplement and HRT in relieving the hot flush as menopausal symptoms in women, it seems that soybean supplements can be an alternative therapy to hormone [20]. Their findings were compatible with our results.

In the recent study we compared the effect of two different doses of piascledine (300 and $600 \mathrm{mg}$ ) with hormone therapy in menopause women. And according to the results, all three groups had a significant reduction in the symptoms related to the menopause.

A significant difference was seen in all groups, so that the hormone therapy had the greatest effect, then in the second group that received piascledine twice a day the symptoms became disappear and finally in the first group received a capsule of piascledine daily the symptoms reduced. The important issue is that the use of piascledine was associated with no complication or side effect such as spotting or vaginal bleeding (the symptoms that we may see in HRT).

The patients using piascledine also doesn't have any concern about the different kinds of cancers related to the hormone therapy, Heart disease or thrombosis so they can confidently use this drug to reduce menopause symptoms.

\section{Conclusion}

We found that a higher dosage of piascledine leads to a significant reduction in hot flushes rate and according to the effectiveness and also lack of side effects related to the higher dose of piascledine, it seems to be an appropriate alternative for hormone replacement therapy to treat the menopausal symptoms in high dosage.

\section{Acknowledgements}

We thank the staffs of Dr. Rasekh clinic for supporting study implementation.

\section{Financial and Competing Interests Disclosure}

The authors report no conflicts of interest.

\section{References}

1. Pronob KD, Manu A. Postmenopausal syndrome. Indian J Psychiatry 2015; 222-232.

2. Farook AA, Santiago P. Hormonal changes during menopause. Maturitas 2009; 135-137.

3. Soules MR, Sherman S, Parrott E, Rebar R, Santoro N, Utian W. Executive summary: Stages of Reproductive Aging Workshop (STRAW). Fertil Steril 2001; 76: 874-878.

4. Sakineh MC, Nazanin R, Sevil H, Ali M, Safoura T, Hamid T, Kourosh S. Quality of life of postmenopausal women and their spouses: a community-based study. Iran Red Crescent Med J 2015; 17: 21599.

5. Joshua Z, Sooyeon A, Claire L, Sara N. Treatment of insomnia, insomnia symptoms, and obstructive sleep apnea during and after menopause: therapeutic approaches. Curr Psychiatry Rev 2015; 63-83.

6. Erin SL, Manisha Desai M, Nancy PJWW. Vitamin D levels and menopause-related symptoms. Menopause 2014; 1197-1203.

7. North American Menopause Society. Treatment of menopause-associated vasomotor symptoms. Position statement of The North American Menopause Society. Menopause 2004; 11-33.

8. Parvaneh M, Faraz M. The effects of valerian root on hot flashes in menopausal women. Iran J Pharm Res 2013; 217-222.

9. Soheila B, Shirin H, Leila FR, Hadi H, Seiedeh HS. The effect of folic acid on menopausal hot flashes: a randomized clinical trial. J Caring Sci 2013; 131-140.

10. Kathryn AM, Robert LB. Treatment of menopausal symptoms with hormone therapy. Lit Rev Curr Thr 2018.

11. Savita RS, Wansalan KS. Comparative study of gabapentin and isoflavone in menopausal vasomotor symptoms. J Midlife Health 2016; 132-139.

12. Min KK, Seok KS, Hee DC, Kyung JH, Tak K, Byung KY, Byung SL. Perceptions of postmenopausal symptoms and treatment options among middle-aged Korean women. Yonsei Med J 2017; 533-539.

13. Solimene MC. Coronary heart disease in women: a challenge for the 21st century. Clinics (Sao Paulo) 2010; 65: 99-106.

14. Da Luz PL, Solimene MC. Terapêutica de reposição hormonal: fim da controvérsia? Rev Bras Med 2003; 60: 337-340.

15. Lewin KJ, Sinclair HK, Bond CM. Womens knowledge of and attitudes towards hormone replacement therapy. Family Pract 2003; 20: 112-119.

16. Mohammad T, An P. Role of phytoestrogens in prevention and management of type 2 diabetes. World $\mathrm{J}$ Diabetes 2015; 271-283.

17. Nair PA. Dermatosis associated with menopause. J Midlife Health 2014; 5: 168-175.

18. Heather BP, Wendy J. The pros and cons of phytoestrogens. Front Neuroendocrinol 2010; 400-419. 
19. Stacie EG, Laura S. Botanical and dietary supplements for menopausal symptoms: what works, what doesnt. J Womens Health (Larchmt) 2007; 634-649.

20. Yunes P, Fatemeh B, Nafise K, Nika BJ, Yahya D. Comparison of piascledine (Avocado and soybean oil) and hormone replacement therapy in menopausal-induced hot flashing. Iran J Pharm Res 2011; 941-951.

21. Ana CM, Ana MS, Maria SS, Vilma AS. Phytoestrogens as alternative hormone replacement therapy in menopause: what is real, what is unknown. J Steroid Biochem Mol Biol 2014; 61-71.

22. Shumaker SA, Legault C, Rapp SR, Thal L, Wallace RB, Ockene JK, Hendrix SL, Jones BN, Assaf AR, Jackson RD, Kotchen JM, Wassertheil-Smoller S, WactawskiWende J, WHIMS Investigators. Estrogen plus progestin and the incidence of dementia and mild cognitive impairment in postmenopausal women: the womens health initiative memory study: a randomized controlled trial. JAMA 2003; 289: 2651-2662.

23. Kim MY, Choi SD, Ryu A. Is complementary and alternative therapy effective for women in the climacteric period? J Menopausal Med 2015; 21: 28-35.

24. Mackey R, Eden J. Phytoestrogens and the menopause. Climacteric 1998; 1: 302-308.

25. Rua I, Centro BR. Phytoestrogens appear to reduce the frequency of hot flushes in menopausal women, without serious side-effects. Afr J Pharm Pharmacol 2014; 1071-1087.

26. Mauviel A, Loyau G, Pujol JP. Effect of unsaponifiable extracts of avocado and soybean (Piasclédine) on the collagenolytic action of cultures of human rheumatoid synoviocytes and rabbit articular chondrocytes treated with interleukin-1. Revue du Rhumatisme et des Maladies Osteo-articulaires 1991; 241-245.
27. Macaigne G, Ozon N, Dikov D, Auriault ML, Deplus R. Piascledine-associated lymphocytic colitis. Gastroenterol Clin Biol 2004; 412-413.

28. Pavelka K, Coste P, Geher P, Krejci G. Efficacy and safety of piascledine 300 versus chondroitin sulfate in a 6 months treatment plus 2 months observation in patients with osteoarthritis of the knee. Clin Rheumatol 2010; 659-670.

29. Cinelli M, Guiducci S, Del Rosso A, Pignone A, Del Rosso M, Fibbi G, Serratì S, Gabrielli A, Giacomelli R, Piccardi N, Matucci Cerinic M. Piascledine modulates the production of VEGF and TIMP-1 and reduces the invasiveness of rheumatoid arthritis synoviocytes. Scand J Rheumatol 2006; 346-350.

30. Laurent GA, Winnie SSC. Osteoarthritis and nutrition. From nutraceuticals to functional foods: a systematic review of the scientific evidence. Arthritis Res Ther 2006; 8: 127.

31. Appelboom T, Schuermans J, Verbruggen G, Henrotin Y, Reginster JY. Symptoms modifying effect of avocado/ soybean unsaponifiables (ASU) in knee osteoarthritis. A double blind, prospective, placebo-controlled study. Scand J Rheumatol ; 242-247.

\section{*Correspondence to}

Zareian Jahromi Mahshid

Jahrom University of Medical Science

Jahrom

Iran 